\title{
Chapter 13 \\ Challenges and Opportunities \\ in Scientific and Technological Support \\ for Monitoring in the Non-proliferation \\ Regime
}

\author{
Jill Hruby
}

This paper focuses largely on the challenges and opportunities for monitoring declared and undeclared facilities for other than peaceful uses. There are five technology areas for monitoring that will be addressed: (1) wide area environmental sampling, (2) open-source satellite imagery, (3) civil society reporting, (4) antineutrino detection, and (5) data fusion and automation to combine information from multiple sources.

Although not covered in this paper, another challenging area for Non-Proliferation Treaty (NPT) monitoring and verification is nuclear weapons dismantlement. This topic is being addressed by the public-private partnership between the U.S. Department of State and the Nuclear Threat Initiative called the International Partnership for Nuclear Disarmament Verification. Monitoring nuclear weapons dismantlement is technically very challenging and there is no silver bullet, but through this effort progress is being made.

Wide Area Environmental Sampling-Techniques for wide area environmental sampling include collection and analysis of atmospheric gases, atmospheric particulates, aquatic materials, vegetation, sediments and soils, and/or fauna. Historically environmental sampling has been deemed too expensive for practical use by the IAEA.

Of the possible wide area sampling techniques, atmospheric sampling has long been thought to be the most effective. Relevant nuclear signatures include Krypton-85 gas as a by-product of Plutonium reprocessing, and Uranyl Fluoride $\left(\mathrm{UO}_{2} \mathrm{~F}_{2}\right)$ aerosol from the interaction of leaked Uranium Hexafluoride $\left(\mathrm{UF}_{6}\right)$ and the air during Uranium enrichment. Reported challenges for atmospheric monitoring include ${ }^{1}:$ the high

\footnotetext{
${ }^{1}$ Wogman [1] and STR-341 from 1995 and re-visit in 2010.

J. Hruby $(\bowtie)$

Nuclear Threat Initiative, Washington, DC, USA

e-mail: jillhruby@gmail.com 
cost of a well populated sampling grid; the difficulty in detecting changes in Krypton85 above baseline even with modeling due to the significant increase in background levels from the growing number of declared reprocessing programs, especially in the Northern Hemisphere ${ }^{2}$; and the sparsity of Uranyl Fluoride generation when improved filtering technologies are incorporated into enrichment plants.

However, there are some important opportunities in atmospheric environmental sampling. One particularly promising area is the use of drones or mobile platforms instead of a larger number of stationary sampling stations. Mobile platforms with miniaturized sensors, real-time data processing, and increasingly sophisticated models provide an opportunity to collect data at different locations with one or a few sampling systems, each responsible for covering numerous grid points. Additionally, the data could be used to optimize sampling locations especially for undeclared facilities or perhaps trigger additional types of sensors to be deployed. Cost savings could come from not having a fixed set of sampling locations, processing fewer samples, and having less labor-intensive analysis.

In addition to drones or mobile platforms, new detection and analyses techniques have also been advancing including especially laser spectroscopy. ${ }^{3}$ Promising work has been done to accurately detect small amounts of Uranyl Fluoride using ultrafast laser filament-induced fluorescence spectroscopy, but not yet solving the cost issue. Techniques like this could become less expensive but also could be selectively used if triggered by mobile platforms, satellite imaging, or civil society reporting.

Additionally, there was work done in the early 2000s in the Central Asia Republics jointly with Sandia Labs, ${ }^{4}$ and a case made by researchers at Idaho National Lab in $2013^{5}$ that suggest aquatic monitoring approaches are worthy of consideration.

The increased sophistication of low-cost small sensors that could be scattered over wide areas with the ability to self-report also represent new opportunities. These sensors would not necessarily be atmospheric samplers, but could be radiation, acoustic, metrological, or other types of sensors that provide triggering data for other monitors, input to models, or corroborative information.

Open-Source Satellite Imagery-The revolution in commercial satellite imaging has provided an incredible boost to nonproliferation monitoring and more opportunities are coming at a rapid pace.

Trends in commercial satellites include larger constellations of smaller satellites with medium to high resolution that image the earth more frequently, and some satellites with very high resolution (less than $50 \mathrm{~cm}$ ) that can stare at specific areas. As an example, Planet Labs has a constellation of 150 satellites in orbit. Their constellation allows line scanning of the entire earth every day with three-meter resolution, and the ability to monitor an area twice a day at $72-\mathrm{cm}$ resolution. Both capabilities have at least 5 years of archived data for comparison.

\footnotetext{
${ }^{2}$ Schoppner et al. [2].

${ }^{3}$ Skrodzki et al. [3].

${ }^{4}$ Passell, H. D. et al., "The Navurz Project: Cooperative, transboundary monitoring, data sharing and modeling of water resources in Central Asia" SAND2006-6673.

${ }^{5}$ Schanfein [4].
} 
New active sensor systems like synthetic aperture $\operatorname{radar}^{6}$ and video imaging allow vehicle movement, among other things, to be tracked. In 2018, the British company Earth-i released the first full-color video of earth from space taken by a commercial satellite. The views include airplanes in motion at Dubai international airport, cars driving in Argentina, and ships leaving port in Norway. Videos like these allow digital surface models to be developed and are useful for urban and remote sites with varied terrain to support inspection activities and to assist in characterizing unknown buildings.

Other new analysis approaches like hyperspectral imaging ${ }^{7}$ can amplify the usefulness of satellite images. Hyperspectral imaging provides the ability to remotely discriminate between elements. European Space Agency's Sentinel-2 satellite now has 12 spectral bands available. Hyperspectral imaging may be particularly useful to identify conversion facilities as well as monitor reprocessing facilities.

New data fusion techniques that combine information from multiple satellites having different sensor suites ${ }^{8}$ is also undergoing revolution. For example, the commercial company Palantir provides sophisticated data fusion services.

Overall, it seems that open source satellite imagery will continue to become more available and less expensive with the ability to get higher resolution images multiple times a day. Video images are also expected to become widely available as is hyperspectral data. Perhaps the largest issue for the nonproliferation community will keeping up with what is available and using it effectively. Caution is needed in using imagery by itself and can lead to conclusions that are not always accurate without additional information or knowledge.

Civil Society Reporting - The phrase civil society reporting is used here to mean engaging volunteers in monitoring, and specifically radiation monitoring in the cases discussed. In general, civil society reporting has been considered for emergency response or incident reporting, however, the use for NPT monitoring could prove promising. As one of the longest and most avid supporters of civil society reporting, Rose Gottemoeller has said "Not for every measure or every treaty would it be appropriate to have citizen volunteers involved. But I think, nevertheless, that in certain settings it could be quite useful to draw them in. And, in certain settings, indeed, dispersed sensing mechanisms on a number of mobile platforms could make a difference to how we understand deployment patterns in the future."9

There are three specific areas of interest to monitoring in the NPT regime, none ready for formal application, but illustrative of possibilities: one is people volunteering to use radiation detectors to record and report doses, two is people using their cell phones as detectors with an affiliated app to record and report radiation doses, and third is social media as a mechanism for reporting and/or obtaining relevant information. Radiation monitoring by volunteers combined with other information could

\footnotetext{
6“Emerging Satellites for Non-Proliferation and Disarmament Verification," Vienna Center for Disarmament and Non-Proliferation, January 2016.

${ }^{7}$ Ziemann and Theiler [5].

${ }^{8}$ Pabian [6].

${ }^{9}$ Goettemoeller [7].
} 
be useful to indicate, for example, a change in normal patterns perhaps indicating the emergence of an undeclared facility or new transportation routes.

A real application of civil society reporting of radiation is Safecast. ${ }^{10}$ Safecast was created after the Fukushima incident to report data that otherwise wasn't available, and now has the world's largest open data set of radiation. A mobile, GPS enabled, logging radiation sensor called bGeigie Nano (the " $b$ " standing for bento box) can be built using on-line instructions, and mounted to the outside of a car, bicycle, train, drone or other mobile platform with data captured on a memory card. Most of the collectors for Safecast are Japanese citizens, but data is being collected worldwide. Recently Safecast began collecting air quality data as well. The willingness of volunteers to collect and post data is an indication of what is possible, and the volunteer nature avoids certain legal issues.

As an alternative to a specialized sensor being built and carried by volunteers, a group of researchers at Idaho National Lab developed the concept of using the CMOS sensor in cell phone cameras to record gamma radiation. ${ }^{11}$ In this approach, the camera lens is covered so that no visible radiation is detected allowing only high energy gamma or X-rays radiation to hit the sensor. If radiation is detected, an app records and transmits the information. Research to fully understand the accuracy and stability of these types of systems is still occurring ${ }^{12}$ and, at the very least, demonstrates the potential of a built-in radiation sensor on a smartphone.

More recently, a research team at North Carolina State University ${ }^{13}$ used commercial surface mount resistors and thermoluminescence to measure natural background radiation levels indicating the potential application beyond elevated emergency response levels. The dose estimates from the resistors can be measured in a lab in hours compared to weeks needed for biodosimetry. Their intended application was separating the worried well from people exposed to radiation after an event, however being able to measure background radiation levels is powerful.

Finally, social media including Twitter, Facebook, YouTube, Flickr, or other platforms represent ubiquitous sources with vast amounts of information distributed quickly. Social media is extensively utilized in emergency events and already Facebook has created a tool called Crisis Response ${ }^{14}$ to respond to a consumer pull. Social media also naturally results in a cueing mechanism for others to collect and post data in regions of interest.

Social media for treaty monitoring is a more nuanced issue, and social media for verification even more so. It is conceivable that social media could be used as another open source data feed for wide are monitoring, especially in combination with other data for corroboration. The idea of using social media in treaties could be included in monitoring and verification provisions. ${ }^{15}$

\footnotetext{
${ }^{10}$ blog.safecast.org, accessed October 9, 2019.

${ }^{11}$ Cogliati et al. [8].

${ }^{12}$ Van Hoey et al. [9].

${ }^{13}$ Hayes and O'Mara [10].

${ }^{14} \mathrm{https}: / /$ www.facebook.com/about/crisisresponse/, accessed October 9, 2019.

${ }^{15}$ Lorenz and Feldman [11] and Bufford [12].
} 
Anti-Neutrino Detection - In addition to the technologies already discussed, antineutrino detection is an important monitoring approach especially for reactors of key importance. Antineutrino detectors offer a potential solution for continuous, real-time verification of nuclear reactor operation without having to be in the reactor core. The flux of antineutrinos that leaves a reactor carries information about the reactor power and the fissile material inventory, both important for safeguards.

While neutrino detection was discovered in the 1950 s, the experiments to understand neutrino oscillation in liquid scintillators that occurred in the 1990s demonstrated much of the physics required for reactor monitoring. Since about 2000, development of detectors that would operate within a few tens of meters from the reactor core has been on-going.

In 2008, IAEA held a workshop on antineutrino detection for safeguards applications that concluded antineutrino detectors have unique abilities to non-intrusively monitor reactor operational status, power, and fissile content in real time, from outside containment. ${ }^{16}$ The optimized neutrino detector for reactor monitoring and safeguards would be relatively compact in size and preferably movable. Today, the most likely scenario for antineutrino based cooperative monitoring would be the deployment of a cubic meter scale detector relatively near the reactor. Longer range monitoring is also attractive but requires technical advances. On-going work on neutrino detection includes segmented photomultiplier detectors ${ }^{17}$ and high-fidelity computer simulations. ${ }^{18}$

Data Fusion and Automation-In order to use data from many different types of sensors, data management and fusion are key. Layers of complexity emerge as sensor data is combined with imagery and social media and as verification is required.

As an example of data fusion and analysis challenges and opportunities, a project is being funded by the United States National Nuclear Security Administration called MINOS-Multi-Informatics for Nuclear Operations Scenarios. ${ }^{19}$ In this project, data is being collected on nuclear operations at Oak Ridge National Laboratory where ground truth is available. Different detectors and sensors including radiation, acoustic, seismic, biota, and imagery are being collected and data management and data analytics are then used to draw conclusions about the information to compare with ground truth information. The goal of the work is to see if a highly automated system can answer the question of whether special nuclear material is being diverted from a reactor and is motivated by the large number of declared reactors coming on-line. The project involves ten Department of Energy Laboratories. The data management strategy is to ingest persistent field data; archive, share, and curate the data; and allow analysis to be performed by different groups with flexibility and scalability. The data will include export controlled and Official Use Only information to further

\footnotetext{
${ }^{16}$ Final Report: Focused Workshop on Antineutrino Detection for Safeguards Application, 2830 October 2008, IAEA Headquarters, Vienna, Austria, https://www.lefigaro.fr/assets/pdf/AIEAneutrino.pdf.

${ }^{17}$ Ashenfelter et al. [13].

${ }^{18}$ Stewart et al. [14].

${ }^{19}$ Gaylord [15] and Rajadhyaksha et al. [16].
} 
challenge the data management system. The data ingestion system aims to be entirely automated using domain agnostic user interfaces and multi-petabyte data holdings. The work is just entering the third year of a 7-year planned program.

Summary - The challenge of cost-effectively and comprehensively monitoring in the non-proliferation regime is both large and growing with the number of declared sites, increased background levels, and complex geopolitics. However, simultaneously there is an explosion in ubiquitous sensors, drones and mobile platforms, social media, commercially available satellite imagery, sophisticated and sensitive measurement techniques, new data fusion and data sharing approaches, and interested public citizens that could help with the monitoring challenge. A few examples of opportunities and on-going research have been provided that will be critical for future monitoring. If global support is available to mature these approaches and generate the next generation ideas, then monitoring and verification in the increasingly complex environment can remain.

\section{References}

1. N.A. Wogman, Prospects for the introduction of wide area monitoring using environmental sampling. J. Radiol. Nucl. Chem. 296(2), 1071-1077 (2012)

2. M. Schoppner, M. Walker, A. Glaser, Detecting clandestine plutonium separation activities with Krypton-85, in 56th INMM Meeting, Indian Wells, California, July 2015

3. Skrodzki et al., Ultrafast laser filament-induced fluorescence spectroscopy of uranyl fluoride. Sci. Rep. 8, 11629 (2018)

4. M. Schanfein, The case for the application of worldside marine radioactivity studies in the search for undeclared facilities and activities. Institute of Nuclear Materials Management, July 2013

5. A. Ziemann, J. Theiler, Material detection in hyperspectral imagery in support of nuclear nonproliferation, in Advances in Nuclear Nonproliferation Technology and Policy Conference: Bridging the Gaps in Nuclear Nonproliferation, ANTPC 2016, 2016

6. F. Pabian, Commercial satellite imagery as an evolving open-source verification technology: emerging trends and their impact for nuclear nonproliferation analysis. JRC Technical Reports (2015)

7. R. Goettemoeller, NATO Nuclear Policy in a Post-INF. World University of Oslo, 09 Sept 2019

8. J. Cogliati, K. Derr, J. Wharton, Using CMOS sensors in a cellphone for gamma detection and classification. eprint arXiv:1401.0766 [physics.ins-det], Jan 2014

9. O. Van Hoey et al., Radiation dosimetry properties of smartphone CMOS sensors. Radiat. Prot. Dosim. 168(3), 314-321 (2016)

10. R.B. Hayes, R.P. O'Mara, Retrospective dosimetry at the natural background level with commercial surface mount resistors. Radiat. Meas. 121, 42-48 (2019)

11. T. Lorenz, Y. Feldman, The efficacy of social media as a research tool and information source for safeguards verification, in Information Analysis Technologies, Techniques and Methods for Safeguards, Nonproliferation and Arms Control Verification Workshop. Workshop Proceedings, Portland, OR, 12-14 May 2014

12. J. Bufford, Societal verification: past and present, in Information Analysis Technologies, Techniques and Methods for Safeguards, Nonproliferation and Arms Control Verification Workshop. Workshop Proceedings, Portland, OR, 12-14 May 2014

13. J. Ashenfelter et al., The PROSPECT physics program. J. Instrum. 11, P11004 (2015) 
14. C. Stewart et al., Employing antineutrino detectors to safeguard future nuclear reactors from diversions. Nat. Commun. 10, 3527 (2019). https://doi.org/10.1038/s41467-019-11434-z. www.nature.com/naturecommunication

15. J. Gaylord, Data management systems for monitoring workloads, in Data Over Distance Symposium, 19 July 2018

16. A. Rajadhyaksha et al., Multi-informatics for nuclear operations scenarios, in Consortium for Verification Technology Workshop, 31 Oct to 1 Nov 2018

Open Access This chapter is licensed under the terms of the Creative Commons Attribution 4.0 International License (http://creativecommons.org/licenses/by/4.0/), which permits use, sharing, adaptation, distribution and reproduction in any medium or format, as long as you give appropriate credit to the original author(s) and the source, provide a link to the Creative Commons license and indicate if changes were made.

The images or other third party material in this chapter are included in the chapter's Creative Commons license, unless indicated otherwise in a credit line to the material. If material is not included in the chapter's Creative Commons license and your intended use is not permitted by statutory regulation or exceeds the permitted use, you will need to obtain permission directly from the copyright holder. 